\title{
Robust Control Design of Uncertain Discrete-Time Descriptor Systems with Delays
}

\author{
Jun Yoneyama, Yuzu Uchida and Ryutaro Takada \\ Additional information is available at the end of the chapter
}

http://dx.doi.org/10.5772/51538

\section{Introduction}

A descriptor system describes a natural representation for physical systems. In general, the continuous-time descriptor representation consists of differential and algebraic equations, and the discrete-time descriptor system has difference and algebraic equations. Hence, the descriptor system is a generalized representation of the state-space system. This system appears in various physical systems. In fact, descriptor systems can be found in electrical circuits, moving robots and many other practical systems which are modeled with additional algebraic constraints. The descriptor system is also referred to as singular system, implicit system, generalized state-space system, differential-algebraic system, or semistate system. System analysis and control design of descriptor systems have been extensively investigated in the past years due to their potential representation ([4], [6], [7], [17], [23]). An important characteristic of continuous-time descriptor systems is the possible impulse modes, which are harmful to physical systems and are undesirable in system control. The discrete-time descriptor system may not have causality, which leads to no solution of the system states. In [4], [32], such descriptor system behaviors are described and notion of regularity, non-impulse, causality, and admissibility are given. In [1] and [22], quadratic stability for continuous-time descriptor systems was considered. Its discrete-time system counterpart was investigated in [31] and [32].

When we make a mathematical model for a physical system, time-delay is another phenomenon. We often see time-delay in the process of control algorithms and the transmission of information. Time-delay often appear in many practical systems and mathematical formulations such as electrical system, mechanical system, biological system, and transportation system. Hence, a system with time-delay is also a natural representation, and its analysis and synthesis are of theoretical and practical importance. In the past decades, research on continuous-time delay systems has been active. Difficulty that arises in continuous time-delay systems is that the system is infinite dimensional and a corresponding controller can be a memory feedback. This class of a controller may minimize a certain performance index, but it is difficult to implement it to practical systems because it feeds back past information of the system. To overcome such a difficulty, a memoryless controller 
is used for time-delay systems. In the last decade, sufficient stability conditions for time-delay systems have been given via linear matrix inequalities (LMIs), and stabilization methods by memoryless controllers have been investigated by many researchers. Since Li and de Souza considered robust stability and stabilization problems in [18], less conservative stability conditions for continuous time-delay systems have been obtained in [14] and [26]. Recently, $\mathrm{H}_{\infty}$ disturbance attenuation conditions have also been given ([25], [34], [35]). The results in [10], [27], [33], [36] considered discrete-time systems with time-invariant delays. Gao and Chen [11], Hara and Yoneyama [12], [13] gave robust stability conditions. Fridman and Shaked [8] solved a guaranteed cost control problem. Fridman and Shaked [9], Zhang and Han [37] considered the $\mathrm{H}_{\infty}$ disturbance attenuation. The results have been extended to a class of discrete-time descriptor delay systems in [2], [3], [24].

In general, control systems are designed not only for the stability, but also for robustness with respect to system parameters. In addition, they are designed for the optimization of multiple control performance measures. Most designed control systems require accurate controllers. Thus, when a desired controller is implemented, all of the controller coefficients are required to be the exact values as those to be designed. However, it is not always possible in practical applications since actuators may be of malfunction, and round-off errors in numerical computations by calculations are possibly encountered. Therefore, it is necessary that the designed controller should be able to tolerate some uncertainty in its control gains. Since controller fragility problem has to be considered when implementing a designed controller in practical applications, the non-fragile control design problem has been investigated in [5], [15], [16], [19], [20], [21]. For state-space systems, several recent research works have been devoted to the design problem of non-fragile robust control ([5], [19], [20], [21]). Most of these are derived via either Riccati matrix equation approach or linear matrix inequality (LMI) approach. The design problem of non-fragile robust controllers of continuous-time descriptor systems was investigated in [15] and [16]. The discrete-time counterpart was given in [28].

In this chapter, the robust non-fragile control design problem and the robust $\mathrm{H}_{\infty}$ non-fragile control design problem for uncertain discrete-time descriptor systems are considered. The controller gain uncertainties and uncertain system parameters under consideration are supposed to be time-varying but norm-bounded. The problem to be addressed is the control design problem of state feedback controller, which is subject to norm-bounded uncertainty, such that the resulting closed-loop system is regular, causal and robustly admissible with $\mathrm{H}_{\infty}$ disturbance attenuation for all admissible uncertainties. Sufficient conditions for the solvability of the robust $\mathrm{H}_{\infty}$ non-fragile control design problem for descriptor systems are obtained, for the cases with multiplicative controller uncertainties. The results are developed for a class of uncertain discrete-time descriptor systems with time-delay. Finally, some numerical examples are shown to illustrate our proposed controller design methods.

\section{Descriptor systems}

Consider the discrete-time descriptor system

$$
E x(k+1)=A x(k)+B u(k)
$$


where $x(k) \in \Re^{n}$ is the state and $u(k) \in \Re^{m}$ is the control. $E, A$ and $B$ are system matrices with appropriate dimensions. $E$ satisfies $r a n k E=r \leq n$. Unforced descriptor system (1) with $u(k)=0$ is denoted by the pair $(E, A)$.

Definition 2.1. (Dai [4]) (i) The pair $(E, A)$ is said to be regular if $\operatorname{det}(z E-A)$ is not identically zero.

(ii) The pair $(E, A)$ is said to be causal if it is regular and $\operatorname{deg}(\operatorname{det}(z E-A))=\operatorname{rank}(E)$.

(iii) Define the generalized spectral radius as $\rho(E, A)=\underset{\lambda \in\{\operatorname{det}(z E-A)=0\}}{\max }|\lambda|$. The pair $(E, A)$ is said to be stable if $\rho(E, A)<1$.

(iv) The pair $(E, A)$ is said to be admissible if it is regular, causal and stable.

Lemma 2.2. (Dai [4]) (i) The descriptor system

$$
E x(k+1)=A x(k)
$$

where

$$
(E, A)=\left(\left[\begin{array}{cc}
I_{r} & 0 \\
0 & 0
\end{array}\right],\left[\begin{array}{ll}
A_{1} & A_{2} \\
A_{3} & A_{4}
\end{array}\right]\right)
$$

is regular and causal if and only if $A_{4}$ is invertible.

(ii) The pair $(E, A)$ is admissible if and only if $A_{4}$ is nonsingular and $\rho\left(A_{1}-A_{2} A_{4}^{-1} A_{3}\right)<1$.

Unlike the standard state-space system, a descriptor system may not be regular and causal. These unique characteristics lead to no solution of the system (1), and thus should be taken care of.

Next, we consider the uncertain descriptor system

$$
E x(k+1)=(A+\Delta A) x(k)+B u(k)
$$

where an uncertain matrix is of the form

$$
\Delta A=H F(k) G
$$

where $F(k) \in \Re^{l \times j}$ is an unknown time-varying matrix satisfying $F^{T}(k) F(k) \leq I$ and $H$ and $G$ are constant matrices of appropriate dimensions.

\section{Non-fragile control}

This section provides system analysis and control design for uncertain descriptor systems. First, the non-fragile controller is discussed in Section 3.1, and then the system analysis of the closed-loop system with a non-fragile controller is investigated in Section 3.2. Finally, Section 3.3 proposes non-fragile control design methods. 


\subsection{Form of controller and preliminary results}

The ideal form of a feedback controller is given by

$$
u(k)=K x(k)
$$

where $K$ is a feedback gain to be determined. In practical situations where malfunction in the actuator and uncertain calculation of control gain may occur, the actual controller is assumed to be of the form

$$
u(k)=[I+\alpha \Phi(k)] K x(k)
$$

where $\alpha \Phi(k) K$ shows uncertainty in the control gain. $\Phi(k)$ is an unknown time-varying matrix satisfying

$$
\Phi^{T}(k) \Phi(k) \leq I
$$

and $\alpha$ is a known positive constant which indicates the measure of non-fragility against controller gain variation. Applying the controller (4) to the system (2), we have the closed-loop system

$$
E x(k+1)=\left(A+B K+H_{c} F_{c}(k) G_{c}\right) x(k)
$$

where

$$
H_{c}=\left[\begin{array}{ll}
H & \alpha B
\end{array}\right], F_{c}(k)=\operatorname{diag}[F(k) \Phi(k)], G_{c}=\left[\begin{array}{l}
G \\
K
\end{array}\right] .
$$

Definition 3.1. The system (6) is said to be robustly admissible if it is admissible for all admissible uncertainties (3) and (5).

The problem is to find a controller (4) which makes the system (2) robustly admissible. In the following, we consider the robust admissibility of the closed-loop system (6). The following lemmas are useful to prove our results.

Lemma 3.2. (Xie [29]) Given matrices $Q=Q^{T}, H, G$ and $R=R^{T}>0$ with appropriate dimensions.

$$
Q+H F(k) G+G^{T} F^{T}(k) H^{T}<0
$$

for all $F(k)$ satisfying $F^{T}(k) F(k) \leq R$ if and only if there exists a scalar $\varepsilon>0$ such that

$$
Q+\frac{1}{\varepsilon} H H^{T}+\varepsilon G^{T} R G<0
$$


Lemma 3.3. (Xu \& Lam [32]) (i) The descriptor system (1) is admissible if and only if there exist matrices $P>0$ and $Q$ such that

$$
A^{T} P A-E^{T} P E+A^{T} S Q^{T}+Q S^{T} A<0
$$

where $S \in \Re^{n \times(n-r)}$ is any matrix with full column rank and satisfies $E^{T} S=0$.

(ii) The descriptor system (1) is admissible if and only if there exists a matrix $P$ such that

$$
\begin{array}{r}
E^{T} P E \geq 0, \\
A^{T} P A-E^{T} P E<0 .
\end{array}
$$

Lemma 3.4. (Xie and de Souza [30]) Given matrices $X, Y>0$ and $Z$ with appropriate dimensions, we have

$$
X^{T} Z+Z^{T} X+X^{T} Y X \geq-Z^{T} Y^{-1} Z
$$

\subsection{Robust admissibility analysis}

The following two theorems give a necessary and sufficient condition for the closed-loop system (6) to be robustly admissible.

Theorem 3.5. Given $K$, the descriptor system (6) is robustly admissible if and only if there exist matrices $P>0, Q$ and scalar $\varepsilon>0$ such that

$$
\left[\begin{array}{cccc}
Q S^{T}(A+B K)+(A+B K)^{T} S Q^{T} & & & \\
-E^{T} P E+\varepsilon\left(G^{T} G+K^{T} K\right) & (A+B K)^{T} P & Q S^{T} H & \alpha Q S^{T} B \\
P(A+B K) & -P & P H & \alpha P B \\
H^{T} S Q^{T} & H^{T} P & -\varepsilon I & 0 \\
\alpha B^{T} S Q^{T} & \alpha B^{T} P & 0 & -\varepsilon I
\end{array}\right]<0
$$

where $S \in \Re^{n \times(n-r)}$ is any matrix with full column rank and satisfies $E^{T} S=0$.

Proof: (Sufficiency) Suppose that there exist matrices $P>0, Q$ and scalar $\varepsilon>0$ such that the condition (8) holds. Then, by Schur complement formula, we have

$$
\begin{aligned}
& {\left[\begin{array}{c}
Q S^{T}(A+B K)+(A+B K)^{T} S Q^{T}-E^{T} P E(A+B K)^{T} P \\
-P
\end{array}\right]} \\
& +\varepsilon^{-1}\left[\begin{array}{c}
Q S^{T} H_{c} \\
P H_{c}
\end{array}\right]\left[\begin{array}{c}
\left.H_{c}^{T} S Q^{T} H_{c}^{T} P\right]+\varepsilon\left[\begin{array}{c}
G_{c}^{T} \\
0
\end{array}\right]\left[\begin{array}{ll}
G_{c} & 0
\end{array}\right]<0 .
\end{array}\right.
\end{aligned}
$$


Now, using Lemma 3.2, we have

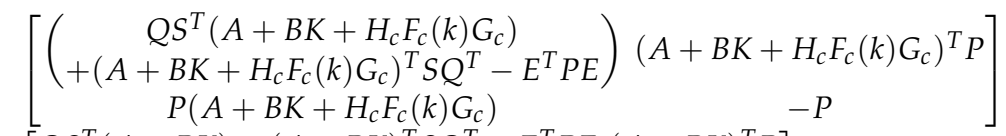

$$
\begin{aligned}
& =\left[\begin{array}{c}
Q S^{T}(A+B K)+(A+B K)^{T} S Q^{T}-E^{T} P E(A+B K)^{T} P \\
P(A+B K)
\end{array}\right] \\
& +\left[\begin{array}{c}
Q S^{T} H_{c} \\
P H_{c}
\end{array}\right] \Phi(k)\left[\begin{array}{ll}
G_{c} & 0
\end{array}\right]+\left[\begin{array}{c}
G_{c}^{T} \\
0
\end{array}\right] \Phi(k)\left[H_{c}^{T} S Q^{T} H_{c}^{T} P\right] \\
& \leq\left[\begin{array}{c}
Q S^{T}(A+B K)+(A+B K)^{T} S Q^{T}-E^{T} P E(A+B K)^{T} P \\
P(A+B K) \\
-P
\end{array}\right] \\
& \varepsilon^{-1}\left[\begin{array}{c}
Q S^{T} H_{c} \\
P H_{c}
\end{array}\right]\left[H_{c}^{T} S Q^{T} H_{c}^{T} P\right]+\varepsilon\left[\begin{array}{c}
G_{c}^{T} \\
0
\end{array}\right]\left[\begin{array}{ll}
G_{c} & 0
\end{array}\right] .
\end{aligned}
$$

It follows from (9) that

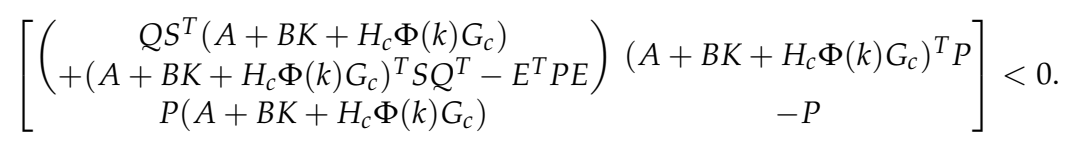

This implies by Schur complement formula and Lemma 3.3(i) that the descriptor system (6) is robustly admissible.

(Necessity) Assume that the descriptor system (6) is robustly admissible. Then, it follows from Definition 3.1 and Lemma 3.3 that there exist matrices $P>0, Q$ and scalar $\varepsilon>0$ such that (7) with $A$ replaced by $A+B K+H_{c} \Phi(k) G_{c}$ holds. Thus, for all admissible $F(k)$ and $\Phi(k)$, the following inequality holds:

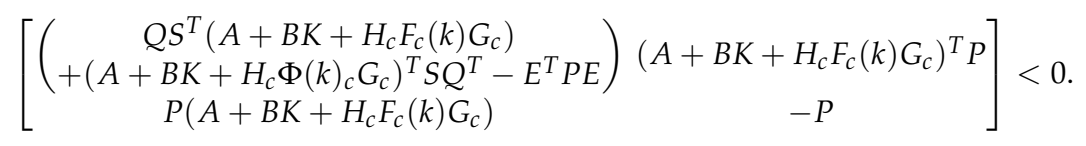

That is,

$$
\begin{gathered}
{\left[\begin{array}{c}
Q S^{T}(A+B K)+(A+B K)^{T} S Q^{T}-E^{T} P E(A+B K)^{T} P \\
P(A+B K)
\end{array}\right]} \\
+\left[\begin{array}{c}
Q S^{T} H_{c} \\
P H_{c}
\end{array}\right] \Phi(k)\left[\begin{array}{ll}
G_{c} & 0
\end{array}\right]+\left[\begin{array}{c}
G_{c}^{T} \\
0
\end{array}\right] \Phi(k)\left[H_{c}^{T} S Q^{T} H_{c}^{T} P\right]<0
\end{gathered}
$$

is satisfied for all admissible $F(k)$ and $\Phi(k)$. It follows from Lemma 3.2 that

$$
\begin{gathered}
{\left[\begin{array}{c}
Q S^{T}(A+B K)+(A+B K)^{T} S Q^{T}-E^{T} P E(A+B K)^{T} P \\
-P
\end{array}\right]} \\
+\varepsilon^{-1}\left[\begin{array}{c}
Q S^{T} H_{c} \\
P H_{c}
\end{array}\right]\left[\begin{array}{c}
\left.H_{c}^{T} S Q^{T} H_{c}^{T} P\right]+\varepsilon\left[\begin{array}{c}
G_{c}^{T} \\
0
\end{array}\right]\left[\begin{array}{ll}
G_{c} & 0
\end{array}\right]<0,
\end{array}\right.
\end{gathered}
$$

which leads to the condition (8) by Schur complement formula. This completes the proof. 
Similarly, using Lemma 3.3(ii), we obtain the following result.

Theorem 3.6. Given $K$, the descriptor system (6) is robustly admissible if and only if there exist matrix $P$ and scalar $\varepsilon>0$ such that

$$
\begin{aligned}
& E^{T} P E \geq 0, \\
& {\left[\begin{array}{cccc}
-E^{T} P E+\varepsilon\left(G^{T} G+K^{T} K\right) & (A+B K)^{T} P & 0 & 0 \\
P(A+B K) & -P & P H & \alpha P B \\
0 & H^{T} P & -\varepsilon I & 0 \\
0 & \alpha B^{T} P & 0 & -\varepsilon I
\end{array}\right]<0 .}
\end{aligned}
$$

\subsection{Robust control design}

In the previous section, we have obtained robust admissibility conditions of the closed-loop system (6). Based on those conditions, we now seek how to calculate a feedback gain $K$ in the controller (4).

Theorem 3.7. There exists a controller (4) that makes the descriptor system (2) robustly admissible if there exist matrices $P>0, Q$ and scalar $\varepsilon>0$ such that

$$
\begin{gathered}
\Gamma=P^{-1}-\varepsilon^{-1} H_{c} H_{c}^{T}>0, \\
Q S^{T} A+A^{T} S Q^{T}-E^{T} P E+\varepsilon G^{T} G+\varepsilon^{-1} Q S^{T} H_{c} H_{c}^{T} S Q^{T}+\Theta^{T} \Gamma^{-1} \Theta-\Psi \Lambda^{-1} \Psi^{T}<0
\end{gathered}
$$

where $S \in \Re^{n \times(n-r)}$ is any matrix with full column rank and satisfies $E^{T} S=0$, and

$$
\begin{aligned}
& \Psi=Q S^{T} B+\Theta^{T} \Gamma^{-1} B, \\
& \Lambda=B^{T} \Gamma^{-1} B+\varepsilon I \\
& \Theta=A+\varepsilon^{-1} H_{c} H_{c}^{T} S Q^{T} .
\end{aligned}
$$

In this case, a feedback gain in the controller (4) is given

$$
K=-\Lambda^{-1} \Psi^{T}
$$

Proof: (Sufficiency) The closed-loop system (6) with the feedback gain (14) is given by

$$
E x(k+1)=\left(A-B \Lambda^{-1} \Gamma^{T}+H_{\mathcal{C}} F_{\mathcal{c}}(k) G_{\mathcal{c}}\right) x(k) .
$$

where $G_{c}=\left[G^{T}-\Psi \Lambda^{-1}\right]^{T}$. Then, by some mathematical manipulation and (13), it can be verified that 


$$
\begin{aligned}
& Q S^{T}\left(A-B \Lambda^{-1} \Psi^{T}\right)+\left(A-B \Lambda^{-1} \Psi^{T}\right)^{T} S Q^{T}-E^{T} P E+\varepsilon G_{c}^{T} G_{c}+\varepsilon^{-1} Q S^{T} H_{c} H_{c}^{T} S Q^{T} \\
& +\left(A-B \Lambda^{-1} \Psi^{T}+\varepsilon^{-1} H_{c} H_{c}^{T} S Q^{T}\right)^{T} \Gamma^{-1}\left(A-B \Lambda^{-1} \Psi^{T}+\varepsilon^{-1} H_{c} H_{c}^{T} S Q^{T}\right) \\
= & Q S^{T} A+A^{T} S Q^{T}-E^{T} P E+\left(A+\varepsilon^{-1} H_{c} H_{c}^{T} S Q^{T}\right)^{T} \Gamma^{-1}\left(A+\varepsilon^{-1} H_{c} H_{c}^{T} S Q^{T}\right) \\
& +\varepsilon G^{T} G-Q S^{T} B \Lambda^{-1} \Psi^{T}-\left(A+\varepsilon^{-1} H_{c} H_{c}^{T} S Q^{T}\right)^{T} \Gamma^{-1} B \Lambda^{-1} \Psi^{T}-\Psi \Lambda^{-1} B^{T} S Q^{T} \\
& -\Psi \Lambda^{-1} B^{T} \Gamma^{-1}\left(A+\varepsilon^{-1} H_{c} H_{c}^{T} S Q^{T}\right)+\Psi \Lambda^{-1} B^{T} \Gamma^{-1} B \Lambda^{-1} \Gamma^{T}+\varepsilon \Psi \Lambda^{-1} \Lambda^{-1} \Psi^{T} \\
& +\varepsilon^{-1} Q S^{T} H_{c} H_{c}^{T} S Q^{T} \\
= & Q S^{T} A+A^{T} S Q^{T}-E^{T} P E+\left(A+\varepsilon^{-1} H_{c} H_{c}^{T} S Q^{T}\right)^{T} \Gamma^{-1}\left(A+\varepsilon^{-1} H_{c} H_{c}^{T} S Q^{T}\right) \\
& +\varepsilon G^{T} G-\left[Q S^{T}+\left(A+\varepsilon^{-1} H_{c} H_{c}^{T} S Q^{T}\right)^{T} \Gamma^{-1}\right] B \Lambda^{-1} \Psi^{T} \\
& -\Psi \Lambda^{-1} B^{T}\left[S Q^{T}+\Gamma^{-1}\left(A+\varepsilon^{-1} H_{c} H_{c}^{T} S Q^{T}\right)\right]+\Psi \Lambda^{-1} \Psi^{T}+\varepsilon^{-1} Q S^{T} H_{c} H_{c}^{T} S Q^{T} \\
= & Q S^{T} A+A^{T} S Q^{T}-E^{T} P E+\varepsilon G^{T} G+\varepsilon^{-1} Q S^{T} H_{c} H_{c}^{T} S Q^{T}+\Theta^{T} \Gamma^{-1} \Theta-\Psi \Lambda^{-1} \Psi^{T}<0 .
\end{aligned}
$$

By Schur complement formula, we obtain

$$
\left[\begin{array}{ccc}
Q S^{T}\left(A-B \Lambda^{-1} \Psi^{T}\right)+\left(A-B \Lambda^{-1} \Psi^{T}\right)^{T} S Q^{T} & & \\
-E^{T} P E+\varepsilon G^{T} G+\varepsilon \Psi \Lambda^{-1} \Lambda^{-1} \Psi^{T} & \left(A-B \Lambda^{-1} \Psi^{T}\right)^{T} P & Q S^{T} H_{\mathcal{c}} \\
P\left(A-B \Lambda^{-1} \Psi^{T}\right) & -P & P H_{\mathcal{c}} \\
H_{c}^{T} S Q^{T} & H_{c}^{T} P & -\varepsilon I
\end{array}\right]<0 .
$$

Hence, by Theorem 3.5 we can show that the closed-loop system (6) is robustly admissible. (Necessity) Assume there exists a feedback control of the form (4) which makes the descriptor system (1) robustly admissible. Then, it follows from Theorem 3.5 that there exists a scalar $\varepsilon>0$ such that

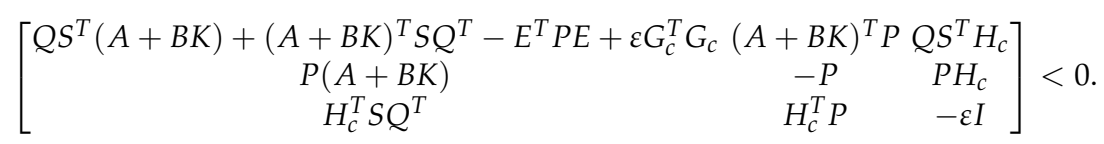

It follows from Schur complement formula that

$$
Q S^{T} A+A^{T} S Q^{T}-E^{T} P E+\varepsilon^{-1} Q S^{T} H_{c} H_{c}^{T} S Q^{T}+\Theta^{T} \Gamma^{-1} \Theta+K^{T} \Psi^{T}+\Psi K+K^{T} \Lambda K<0 .
$$

By Lemma 3.4, we have

$$
K^{T} \Psi^{T}+\Psi K+K^{T} \Lambda K \geq-\Psi \Lambda^{-1} \Psi^{T} .
$$

Therefore, we obtain the conditions (12) and (13), and the feedback gain $K$ is calculated as in (14).

Similarly, we can prove the following theorem by using Theorem 3.6.

Theorem 3.8. There exists a controller (4) that makes the descriptor system (1) robustly admissible if there exist matrix $P$ and scalar $\varepsilon>0$ such that (10), (12), and

$$
-E^{T} P E+\varepsilon G^{T} G+A^{T} \Gamma^{-1} A-A^{T} \Gamma^{-1} B \Lambda^{-1} B^{T} \Gamma^{-1} A<0
$$

where $\Gamma, \Lambda$ are given in Theorem 3.7. In this case, the feedback gain in the controller (4) is given as in (14). 


\section{4. $\mathrm{H}_{\infty}$ non-fragile control}

In this section, we consider the robust admissibility with $\mathrm{H}_{\infty}$ disturbance attenuation. $\mathrm{H}_{\infty}$ disturbance attenuation problem plays an important role in control systems. Section 4.1 discusses the analysis of $\mathrm{H}_{\infty}$ disturbance attenuation, and Section 4.2 gives design methods of the $\mathrm{H}_{\infty}$ non-fragile controllers.

\subsection{Robust $\mathrm{H}_{\infty}$ disturbance attenuation for uncertain systems}

First, we consider the robust $\mathrm{H}_{\infty}$ disturbance attenuation $\gamma$ for the following uncertain descriptor system

$$
\begin{aligned}
E x(k+1) & =(A+\Delta A) x(k)+B_{1} w(k)+B_{2} u(k), \\
z(k) & =C x(k)+D u(k)
\end{aligned}
$$

where $w(k) \in \Re^{m_{1}}$ is the disturbances and $z(k) \in \Re^{p}$ is the controlled output. $A, B_{1}, B_{2}, C$ and $D$ are system matrices with appropriate dimensions. Uncertain matrix $\Delta A$ is assumed to be of the form (3).

The controller is assumed to be of the form (4). Applying the controller (4) to the system (16), we have the closed-loop system

$$
\begin{aligned}
E x(k+1) & =\left(A+B_{2} K+H_{c} F_{c}(k) G_{c}\right) x(k)+B_{1} w(k) \\
z(k) & =(C+D K+\alpha D \Phi(k) K) x(k)
\end{aligned}
$$

where

$$
H_{c}=\left[\begin{array}{ll}
H & \alpha B_{2}
\end{array}\right], F_{c}(k)=\operatorname{diag}[F(k) \Phi(k)], G_{\mathcal{C}}=\left[\begin{array}{l}
G \\
K
\end{array}\right]
$$

Define the cost function

$$
J=\sum_{k=0}^{\infty}\left(z^{T}(k) z(k)-\gamma^{2} w^{T}(k) w(k)\right) .
$$

The problem is to find a controller (4) which makes the system (16) with $w(k)=0$ robustly admissible, and makes it satisfy $J<0$ in (18). If there exists such a controller, it is said to be an $\mathrm{H}_{\infty}$ non-fragile controller and the closed-loop system is said to be robustly admissible with $\mathrm{H}_{\infty}$ disturbance attenuation $\gamma$.

The following is a well-known result for the admissibility with $\mathrm{H}_{\infty}$ disturbance attenuation $\gamma$ of linear descriptor systems.

Lemma 4.1. (Xu E Lam [32]) Consider the system

$$
\begin{aligned}
E x(k+1) & =A x(k)+B w(k), \\
z(k) & =C x(k)+D w(k)
\end{aligned}
$$


where $A, B, C$ and $D$ are matrices of appropriate dimensions.

(i) Given a scalar $\gamma>0$. The descriptor system (19) is robustly admissible with $H_{\infty}$ disturbance attenuation $\gamma$ if and only if there exist matrices $P>0, Q$ such that

$$
\left[\begin{array}{cc}
A^{T} P A-E^{T} P E+C^{T} C & A^{T} P B+C^{T} D \\
B^{T} P A+D^{T} C & B^{T} P B+D^{T} D-\gamma^{2} I
\end{array}\right]+\left[\begin{array}{c}
A^{T} \\
B^{T}
\end{array}\right] S Q^{T}+Q S^{T}[A B]<0
$$

where $S \in \Re^{n \times(n-r)}$ is any matrix with full column rank and satisfies $E^{T} S=0$.

(ii) Given a scalar $\gamma>0$. The descriptor system (19) is robustly admissible with $H_{\infty}$ disturbance attenuation $\gamma$ if and only if there exist matrix $P$ such that

$$
\begin{aligned}
& E^{T} P E \geq 0 \\
& {\left[\begin{array}{cc}
A^{T} P A-E^{T} P E+C^{T} C & A^{T} P B+C^{T} D \\
B^{T} P A+D^{T} C & B^{T} P B+D^{T} D-\gamma^{2} I
\end{array}\right]<0 . }
\end{aligned}
$$

The following theorem provides a necessary and sufficient condition for the robust admissibility with $\mathrm{H}_{\infty}$ disturbance attenuation of (17).

Theorem 4.2. Given $\gamma$ and $K$, the descriptor system (17) is robustly admissible with $H_{\infty}$ disturbance attenuation $\gamma$ if and only if there exist matrices $P>0, Q=\left[Q_{1}^{T} Q_{2}^{T}\right]^{T}$ and scalars $\varepsilon_{1}>0, \varepsilon_{2}>0$ such that

$$
\left[\begin{array}{cccccc}
\Pi_{11} & A_{K}^{T} S Q_{2}^{T}+Q_{1} S^{T} B_{1} & A_{K}^{T} P(C+D K)^{T} & Q_{1} S^{T} H_{c} & 0 \\
Q_{2} S^{T} A_{K}+B_{1}^{T} S Q^{T} & \Pi_{22} & B_{1}^{T} P & 0 & Q_{2} S^{T} H_{c} & 0 \\
P A_{K} & P B_{1} & -P & 0 & P H_{c} & 0 \\
C+D K & 0 & 0 & -I & 0 & \alpha D \\
H_{c}^{T} S Q_{1}^{T} & H_{c}^{T} S Q_{2}^{T} & H_{c}^{T} P & 0 & -\varepsilon_{1} I & 0 \\
0 & 0 & 0 & \alpha D^{T} & 0 & -\varepsilon_{2} I
\end{array}\right]<0
$$

where $A_{K}=A+B_{2} K, S \in \Re^{n \times(n-r)}$ is any matrix with full column rank and satisfies $E^{T} S=0$, and

$$
\begin{aligned}
& \Pi_{11}=Q_{1} S^{T} A_{K}-E^{T} P E+A_{K}^{T} S Q_{1}^{T}+\varepsilon_{1} G_{\mathcal{C}}^{T} G_{c}+\varepsilon_{2} K^{T} K \\
& \Pi_{22}=-\gamma^{2} I+Q_{2} S^{T} B_{1}+B_{1}^{T} S Q_{2}^{T} .
\end{aligned}
$$

Proof: (Sufficiency) Suppose that there exist matrices $P>0, Q=\left[\begin{array}{ll}Q_{1}^{T} & Q_{2}^{T}\end{array}\right]^{T}$ and scalars $\varepsilon_{1}>0, \varepsilon_{2}>0$ such that the condition (23) holds. Then, by Schur complement formula, we have

$$
\bar{Q}+\varepsilon_{1}^{-1} \bar{H}_{1} \bar{H}_{1}^{T}+\varepsilon_{1} \bar{G}_{2}^{T} \bar{G}_{1}+\varepsilon_{2}^{-1} \bar{H}_{2} \bar{H}_{2}^{T}+\varepsilon_{2} \bar{G}_{2}^{T} \bar{G}_{2}<0
$$

where 


$$
\begin{aligned}
\bar{Q} & =\left[\begin{array}{cccc}
Q_{1} S^{T} A_{K}-E^{T} P E+A_{K}^{T} S Q_{1}^{T} & A_{K}^{T} S Q_{2}^{T}+Q_{1} S^{T} B_{1} & A_{K}^{T} P(C+D K)^{T} \\
Q_{2} S^{T} A_{K}+B_{1}^{T} S Q^{T} & -\gamma^{2} I+Q_{2} S^{T} B_{1}+B_{1}^{T} S Q_{2}^{T} & B_{1}^{T} P & 0 \\
P A_{K} & P B_{1} & -P & 0 \\
C+D K & 0 & 0 & -I
\end{array}\right], \\
\bar{H}_{1} & =\left[\begin{array}{lll}
H_{c}^{T} S Q_{1}^{T} H_{c}^{T} S Q_{2}^{T} H_{c}^{T} P & 0
\end{array}\right]^{T}, \\
\bar{H}_{2} & =\left[\begin{array}{llll}
0 & 0 & 0 & \alpha D^{T}
\end{array}\right]^{T}, \\
\bar{G}_{1} & =\left[\begin{array}{llll}
G_{c} & 0 & 0 & 0
\end{array}\right], \\
\bar{G}_{2} & =\left[\begin{array}{llll}
K & 0 & 0 & 0
\end{array}\right] .
\end{aligned}
$$

Now, using Lemma 3.2, we have

$$
\begin{aligned}
& {\left[\begin{array}{cccc}
\Delta_{11} & \Delta_{12} & \Delta_{13} & (C+D K+D \Phi(k) K)^{T} \\
\Delta_{12}^{T} & \Delta_{22} & B_{1}^{T} P & 0 \\
\Delta_{13}^{T} & P B_{1} & -P & 0 \\
C+D K+D \Phi(k) K & 0 & 0 & -I
\end{array}\right] } \\
= & \bar{Q}+\bar{H}_{1} F_{c}(k) \bar{G}_{1}+\bar{G}_{1}^{T} F^{T}(k) \bar{H}_{1}^{T}+\bar{H}_{2} \Phi(k) \bar{G}_{2}+\bar{G}_{2}^{T} \Phi^{T}(k) \bar{H}_{2}^{T} \\
\leq & \bar{Q}+\varepsilon_{1}^{-1} \bar{H}_{1} H_{1}^{T}+\varepsilon_{1} \bar{G}_{1}^{T} \bar{G}_{1}+\varepsilon_{2}^{-1} \bar{H}_{2} H_{2}^{T}+\varepsilon_{2} \bar{G}_{2}^{T} \bar{G}_{2}
\end{aligned}
$$

where

$$
\begin{aligned}
& \Delta_{11}=Q_{1} S^{T}\left(A+B_{2} K+H_{c} F_{c}(k) G_{c}\right)+\left(A+B_{2} K+H_{c} F_{c}(k) G_{c}\right)^{T} S Q_{1}^{T}-E^{T} P E, \\
& \Delta_{12}=\left(A+B_{2} K+H_{c} F_{c}(k) G_{c}\right)^{T} S Q_{2}^{T}+Q_{1} S^{T} B_{1}, \\
& \Delta_{13}=\left(A+B_{2} K+H_{c} F_{c}(k) G_{c}\right)^{T} P, \\
& \Delta_{22}=-\gamma^{2} I+Q_{2} S^{T} B_{1}+B_{1}^{T} S Q_{2}^{T} .
\end{aligned}
$$

It follows from (24) that

$$
\left[\begin{array}{cccc}
\Delta_{11} & \Delta_{12} & \Delta_{13} & (C+D K+D \Phi(k) K)^{T} \\
\Delta_{12}^{T} & \Delta_{22} & B_{1}^{T} P & 0 \\
\Delta_{13}^{T} & P B_{1} & -P & 0 \\
C+D K+D \Phi(k) K & 0 & 0 & -I
\end{array}\right]<0 .
$$

This implies by Schur complement formula and Lemma 4.1(i) that the descriptor system (17) is robustly admissible with $\mathrm{H}_{\infty}$ disturbance attenuation $\gamma$.

(Necessity) Assume that the descriptor system (17) is robustly admissible with $\mathrm{H}_{\infty}$ disturbance attenuation $\gamma$. Then, it follows from Lemma 4.1 that there exist matrices $P>0, Q$ and scalars $\varepsilon_{1}>0, \varepsilon_{2}>0$ such that (20) with $A$ replaced by $A+B K+H_{c} F_{c}(k) G_{c}$ and $C$ by $C+D K+\alpha \Phi(k) K$ holds. Thus, for all admissible $F(k)$ and $\Phi(k)$, the following inequality holds:

$$
\left[\begin{array}{cccc}
\Delta_{11} & \Delta_{12} & \Delta_{13} & (C+D K+D \Phi(k) K)^{T} \\
\Delta_{12}^{T} & \Delta_{22} & B_{1}^{T} P & 0 \\
\Delta_{13}^{T} & P B_{1} & -P & 0 \\
C+D K+D \Phi(k) K & 0 & 0 & -I
\end{array}\right]<0 .
$$


That is,

$$
\bar{Q}+\bar{H}_{1} F_{c}(k) \bar{G}_{1}+\bar{G}_{1}^{T} F_{c}^{T}(k) \bar{H}_{1}^{T}+\bar{H}_{2} \Phi(k) \bar{G}_{2}+\bar{G}_{2}^{T} \Phi^{T}(k) \bar{H}_{2}^{T}<0
$$

is satisfied for all admissible $F(k)$ and $\Phi(k)$. It follows from Lemma 3.2 that

$$
\bar{Q}+\varepsilon_{1}^{-1} \bar{H}_{1} \bar{H}_{1}^{T}+\varepsilon_{1} \bar{G}_{1}^{T} \bar{G}_{1}+\varepsilon_{2}^{-1} \bar{H}_{2} \bar{H}_{2}^{T}+\varepsilon_{2} \bar{G}_{2}^{T} \bar{G}_{2}<0,
$$

which leads to the condition (23). This completes the proof.

Based on Lemma 4.1(ii), we have the following theorem. The proof is similar to that of Theorem 4.2, and is thus omitted.

Theorem 4.3. Given $\gamma$ and $K$, the descriptor system (17) is robustly admissible with $H_{\infty}$ disturbance attenuation $\gamma$ if and only if there exist matrix $P$ and scalars $\varepsilon_{1}>0, \varepsilon_{2}>0$ such that (21), and

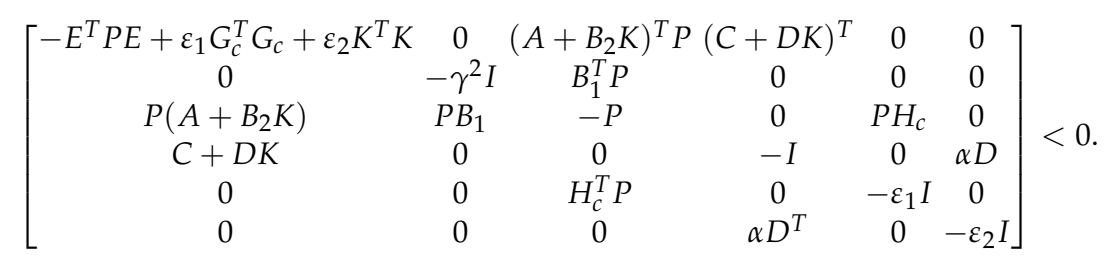

\section{2. $\mathrm{H}_{\infty}$ non-fragile control design for uncertain systems}

Now, we are at the position to propose design methods of $\mathrm{H}_{\infty}$ non-fragile controller for uncertain descriptor systems.

Theorem 4.4. There exists a controller (4) that makes the descriptor system (16) robustly admissible with $H_{\infty}$ disturbance attenuation $\gamma$ if there exist matrices $P>0, Q=\left[\begin{array}{ll}Q_{1}^{T} & Q_{2}^{T}\end{array}\right]^{T}$ and scalars $\varepsilon_{1}>0, \varepsilon_{2}>0$ such that

$$
\begin{array}{r}
\Gamma=P^{-1}-\varepsilon_{1}^{-1} H_{c} H_{c}^{T}>0, \\
Z=I-\varepsilon_{2}^{-1} \alpha^{2} D D^{T}>0, \\
W=\gamma^{2} I-Q_{2} S^{T} B_{1}-B_{1}^{T} S Q_{2}^{T}-\varepsilon_{1}^{-1} Q_{2} S^{T} H_{c} H_{c}^{T} S Q_{2}^{T}-\Theta_{B}^{T} \Gamma^{-1} \Theta_{B}>0, \\
Q_{1} S^{T} A+A^{T} S Q_{1}^{T}-E^{T} P E+\varepsilon_{1} G^{T} G+\varepsilon_{1}^{-1} Q_{1} S^{T} H_{c} H_{c}^{T} S Q_{1}^{T} \\
+C^{T} Z^{-1} C+Y W^{-1} Y^{T}+\Theta_{A}^{T} \Gamma^{-1} \Theta_{A}-\Psi \Lambda^{-1} \Psi^{T}<0
\end{array}
$$

where $S \in \Re^{n \times(n-r)}$ is any matrix with full column rank and satisfies $E^{T} S=0$, and

$$
\begin{aligned}
& \Psi=\left(Q_{1} S^{T}+\Theta_{A}^{T} \Gamma^{-1}\right) B_{2}+C^{T} Z^{-1} D+Y W^{-1}\left(Q_{2} S^{T}+\Theta_{B}^{T} \Gamma^{-1}\right) B_{2}, \\
& \Lambda=B_{2}^{T} \Gamma^{-1} B_{2}+D^{T} Z^{-1} D+\left(\varepsilon_{1}+\varepsilon_{2}\right) I+B_{2}^{T}\left(S Q_{2}^{T}+\Gamma^{-1} \Theta_{B}\right) W^{-1}\left(Q_{2} S^{T}+\Theta_{B}^{T} \Gamma^{-1}\right) B_{2}, \\
& \Theta_{A}=A+\varepsilon_{1}^{-1} H_{c} H_{c}^{T} S Q_{1}^{T}, \\
& \Theta_{B}=B_{1}+\varepsilon_{1}^{-1} H_{c} H_{c}^{T} S Q_{2}^{T}, \\
& Y=A^{T} S Q_{2}^{T}+\left(Q_{1} S^{T}+\Theta_{A}^{T} \Gamma^{-1}\right) \Theta_{B} .
\end{aligned}
$$


In this case, a feedback gain in the controller (4) is given by

$$
K=-\Lambda^{-1} \Psi^{T}
$$

Proof: (Sufficiency) The closed-loop system (17) with the feedback gain (30) is given by

$$
\begin{aligned}
E x(k+1) & =\left(A-B_{2} \Lambda^{-1} \Psi^{T}+H_{c} F_{c}(k) G_{c}\right) x(k)+B_{1} w(k) \\
z(k) & =\left(C-D \Lambda^{-1} \Psi^{T}-\alpha D \Phi(k) \Lambda^{-1} \Psi^{T}\right) x(k)
\end{aligned}
$$

where $G_{\mathcal{C}}=\left[G^{T}-\Psi \Lambda^{-1}\right]^{T}$. Then, by some mathematical manipulation, we have

$$
\begin{aligned}
& Q_{1} S^{T}\left(A-B_{2} \Lambda^{-1} \Psi^{T}\right)+\left(A-B_{2} \Lambda^{-1} \Psi^{T}\right)^{T} S Q_{1}^{T}+\varepsilon_{1} G_{c}^{T} G_{c}+\varepsilon_{2} \Psi \Lambda^{-1} \Lambda^{-1} \Psi^{T} \\
= & Q_{1} S^{T} A-Q_{1} S^{T} B_{2} \Lambda^{-1} \Psi^{T}+A^{T} S Q_{1}^{T}-\Psi \Lambda^{-1} B_{2}^{T} S Q_{1}^{T}+\varepsilon_{1} G^{T} G \\
& +\left(\varepsilon_{1}+\varepsilon_{2}\right) \Psi \Lambda^{-1} \Lambda^{-1} \Psi^{T}, \\
& \left(C-D \Lambda^{-1} \Psi^{T}\right)^{T} Z^{-1}\left(C-D \Lambda^{-1} \Psi^{T}\right) \\
= & C^{T} Z^{-1} C-\Psi \Lambda^{-1} D Z^{-1} C-C^{T} Z^{-1} D \Lambda^{-1} \Psi^{T}+\Psi \Lambda^{-1} D Z^{-1} D \Lambda^{-1} \Psi^{T}, \\
& \left(A-B_{2} \Lambda^{-1} \Psi^{T}+\varepsilon_{1}^{-1} H_{c} H_{c}^{T} S Q_{1}^{T}\right)^{T} \Gamma^{-1}\left(A-B_{2} \Lambda^{-1} \Psi^{T}+\varepsilon_{1}^{-1} H_{c} H_{c}^{T} S Q_{1}^{T}\right) \\
= & \Theta_{A}^{T} \Gamma^{-1} \Theta_{A}-\Psi \Lambda^{-1} B_{2}^{T} \Gamma^{-1} \Theta_{A}-\Theta_{A}^{T} \Gamma^{-1} B_{2} \Lambda^{-1} \Psi^{T}+\Psi \Lambda^{-1} B_{2}^{T} \Gamma^{-1} B_{2} \Lambda^{-1} \Psi T, \\
& {\left[\left(A-B_{2} \Lambda^{-1} \Psi^{T}\right)^{T} S Q_{2}^{T}+\left(Q_{1} S^{T}+\left(A-B_{2} \Lambda^{-1} \Psi^{T}+\varepsilon_{1}^{-1} H_{c} H_{c}^{T} S Q_{1}^{T}\right)^{T} \Gamma^{-1}\right) \Theta_{B}\right] W^{-1} } \\
& \left.\quad \times\left[\left(A-B_{2} \Lambda^{-1} \Psi^{T}\right)^{T} S Q_{2}^{T}+\left(Q_{1} S^{T}+\left(A-B_{2} \Lambda^{-1} \Psi^{T}+\varepsilon_{1}^{-1} H_{c} H_{c}^{T} S Q_{1}^{T}\right)^{T} \Gamma^{-1}\right) \Theta_{B}\right)\right]^{T} \\
= & Y W^{-1} Y^{T}-\Psi \Lambda^{-1} B_{2}^{T}\left(S Q_{2}^{T}+\Gamma^{-1} \Theta_{B}\right)^{T} W^{-1} Y^{T}-Y W^{-1}\left(S Q_{2}^{T}+\Gamma^{-1} \Theta_{B}\right)^{T} B_{2} \Lambda^{-1} \Psi^{T} \\
& +\Psi \Lambda^{-1} B_{2}^{T}\left(S Q_{2}^{T}+\Gamma^{-1} \Theta_{B}\right) W^{-1}\left(S Q_{2}^{T}+\Gamma^{-1} \Theta_{B}\right)^{T} B_{2} \Lambda^{-1} \Psi^{T} .
\end{aligned}
$$

Thus, it can be verified with (29) that

$$
\begin{aligned}
& Q_{1} S^{T}\left(A-B_{2} \Lambda^{-1} \Psi^{T}\right)+\left(A-B_{2} \Lambda^{-1} \Psi^{T}\right)^{T} S Q_{1}^{T}+\varepsilon_{1} G_{c}^{T} G_{c}+\varepsilon_{2} \Psi \Lambda^{-1} \Lambda^{-1} \Psi^{T} \\
& +\left(C-D \Lambda^{-1} \Psi^{T}\right)^{T} Z^{-1}\left(C-D \Lambda^{-1} \Psi^{T}\right)-E^{T} P E+\varepsilon_{1}^{-1} Q_{1} S^{T} H_{c} H_{c}^{T} S Q_{1}^{T} \\
& +\left(A-B_{2} \Lambda^{-1} \Psi^{T}+\varepsilon_{1}^{-1} H_{c} H_{c}^{T} S Q_{1}^{T}\right)^{T} \Gamma^{-1}\left(A-B_{2} \Lambda^{-1} \Psi^{T}+\varepsilon_{1}^{-1} H_{c} H_{c}^{T} S Q_{1}^{T}\right) \\
& +\left[\left(A-B_{2} \Lambda^{-1} \Psi^{T}\right)^{T} S Q_{2}^{T}+\left(Q_{1} S^{T}+\left(A-B_{2} \Lambda^{-1} \Psi^{T}+\varepsilon_{1}^{-1} H_{c} H_{c}^{T} S Q_{1}^{T}\right)^{T} \Gamma^{-1}\right) \Theta_{B}\right] W^{-1} \\
& \left.\times\left[\left(A-B_{2} \Lambda^{-1} \Psi^{T}\right)^{T} S Q_{2}^{T}+\left(Q_{1} S^{T}+\left(A-B_{2} \Lambda^{-1} \Psi^{T}+\varepsilon_{1}^{-1} H_{c} H_{c}^{T} S Q_{1}^{T}\right)^{T} \Gamma^{-1}\right) \Theta_{B}\right)\right]^{T} \\
= & Q_{1} S^{T} A+A^{T} S Q_{1}^{T}+\varepsilon_{1} G^{T} G-E^{T} P E+\varepsilon_{1}^{-1} Q_{1} S^{T} H_{c} H_{c}^{T} S Q_{1}^{T}+C^{T} Z^{-1} C+\Theta_{A}^{T} \Gamma^{-1} \Theta_{A} \\
& +Y W^{-1} Y^{T}-\Psi \Lambda^{-1} \Psi^{T}<0 .
\end{aligned}
$$


By Schur complement formula, we obtain

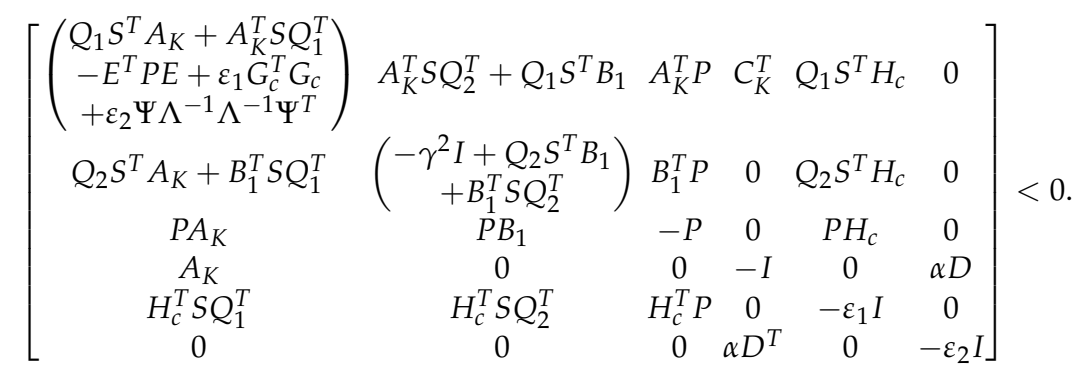

where $A_{K}=A-B_{2} \Lambda^{-1} \Psi^{T}$ and $C_{K}=C-D \Lambda^{-1} \Psi^{T}$. Hence, by Theorem 4.2 we can show that the closed-loop system (17) is robustly admissible with $\mathrm{H}_{\infty}$ disturbance attenuation $\gamma$. (Necessity) Assume there exists a feedback control of the form (4) which makes the descriptor system (16) robustly admissible with $\mathrm{H}_{\infty}$ disturbance attenuation $\gamma$. Then, it follows from Theorem 4.2 that there exist scalars $\varepsilon_{1}>0$ and $\varepsilon_{2}>0$ such that

$$
\left[\begin{array}{cccccc}
\Pi_{11} & A_{K}^{T} S Q_{2}^{T}+Q_{1} S^{T} B_{1} & A_{K}^{T} P(C+D K)^{T} & Q_{1} S^{T} H_{c} & 0 \\
Q_{2} S^{T} A_{K}+B_{1}^{T} S Q^{T} & \Pi_{22} & B_{1}^{T} P & 0 & Q_{2} S^{T} H_{c} & 0 \\
P A_{K} & P B_{1} & -P & 0 & P H_{c} & 0 \\
C+D K & 0 & 0 & -I & 0 & \alpha D \\
H_{c}^{T} S Q_{1}^{T} & H_{c}^{T} S Q_{2}^{T} & H_{c}^{T} P & 0 & -\varepsilon_{1} I & 0 \\
0 & 0 & 0 & \alpha D^{T} & 0 & -\varepsilon_{2} I
\end{array}\right]<0
$$

where

$$
\begin{aligned}
& \Pi_{11}=Q_{1} S^{T} A_{K}+A_{K}^{T} S Q_{1}^{T}-E^{T} P E+\varepsilon_{1} G_{c}^{T} G_{c}+\varepsilon_{2} K^{T} K, \\
& \Pi_{22}=-\gamma^{2} I+Q_{2} S^{T} B_{1}+B_{1}^{T} S Q_{2}^{T} .
\end{aligned}
$$

It follows from Schur complement formula that we obtain the conditions (26), (27), (28), and

$$
\begin{aligned}
& Q_{1} S^{T} A+A^{T} S Q_{1}^{T}-E^{T} P E+\varepsilon_{1} G^{T} G+\varepsilon_{1}^{-1} Q S^{T} H_{c} H_{\mathcal{C}}^{T} S Q^{T}+C^{T} Z^{-1} C+Y W^{-1} Y^{T} \\
& +\Theta_{A}^{T} \Gamma^{-1} \Theta_{A}+K^{T} \Psi^{T}+\Psi K+K^{T} \Lambda K<0 .
\end{aligned}
$$

By Lemma 3.4, we have

$$
K^{T} \Psi^{T}+\Psi K+K^{T} \Lambda K \geq-\Psi \Lambda^{-1} \Psi^{T} .
$$

Therefore, we finally obtain the condition (29), and the feedback gain $K$ as in (30).

Based on Theorem 4.3, we can deduce the following theorem. 
Theorem 4.5. There exists a controller (4) that makes the descriptor system (16) robustly admissible with $H_{\infty}$ disturbance attenuation $\gamma$ if there exist matrix $P$ and scalars $\varepsilon_{1}>0, \varepsilon_{2}>0$ such that (21), (26), (27) and

$$
\begin{array}{r}
W=\gamma^{2} I-B_{1}^{T} \Gamma^{-1} B_{1}>0, \\
-E^{T} P E+\varepsilon_{1} G^{T} G+C^{T} Z^{-1} C+A^{T} \Gamma^{-1} B_{1} W^{-1} B_{1}^{T} \Gamma^{-1} A+A^{T} \Gamma^{-1} A-\Psi \Lambda^{-1} \Psi^{T}<0
\end{array}
$$

where

$$
\begin{aligned}
& \Psi=A^{T} \Gamma^{-1} B_{2}+C^{T} Z^{-1} D+A^{T} \Gamma^{-1} B_{1} W^{-1} B_{1}^{T} \Gamma^{-1} B_{2}, \\
& \Lambda=B_{2}^{T} \Gamma^{-1} B_{2}+D^{T} Z^{-1} D+\left(\varepsilon_{1}+\varepsilon_{2}\right) I+B_{2}^{T} \Gamma^{-1} B_{1} W^{-1} B_{1}^{T} \Gamma^{-1} B_{2} .
\end{aligned}
$$

In this case, a feedback gain in the controller (4) is given as in (30).

\section{Time-delay systems}

This section investigates the robust admissibility of uncertain descriptor delay systems and provides a non-fragile control design method for such systems. Section 5.1 gives a robust admissibility condition for uncertain descriptor delay systems, and Section 5.2 proposes non-fragile control design methods.

\subsection{Robust admissibility for uncertain systems}

Consider the following descriptor system with time-delay and uncertainties:

$$
E x(k+1)=(A+\Delta A) x(k)+\left(A_{d}+\Delta A_{d}\right) x(k-d)+B u(k)
$$

where $x(k) \in \Re^{n}$ is the state and $u(k) \in \Re^{m}$ is the control. $A, A_{d}$ and $B$ are system matrices with appropriate dimensions. $d$ is a constant delay and may be unknown. Uncertain matrices are given by

$$
\left[\Delta A \Delta A_{d}\right]=H F(k)\left[G G_{d}\right]
$$

where $F(k) \in \Re^{l \times j}$ is an unknown time-varying matrix satisfying $F^{T}(k) F(k) \leq I$ and $H$, $G$ and $G_{d}$ are constant matrices of appropriate dimensions. Unforced nominal descriptor system (34) with $u(k)=0$ and $\Delta A=\Delta A_{d}=0$ is denoted by the triplet $\left(E, A, A_{d}\right)$.

Definition 5.1. (i) The triplet $\left(E, A, A_{d}\right)$ is said to be regular if $\operatorname{det}\left(z^{d+1} E-z^{d} A-A_{d}\right)$ is not identically zero.

(ii) The triplet $\left(E, A, A_{d}\right)$ is said to be causal if it is regular and $\operatorname{deg}\left(z^{n d} \operatorname{det}\left(z E-A-z^{d} A_{d}\right)\right)=$ nd $+\operatorname{rank}(E)$.

(iii) Define the generalized spectral radius as $\rho\left(E, A, A_{d}\right)=\max _{z \mid \lambda \in\left\{\operatorname{det}\left(z^{d+1} E-z^{d} A-A_{d}\right)=0\right\}}|\lambda|$. The triplet $\left(E, A, A_{d}\right)$ is said to be stable if $\rho\left(E, A, A_{d}\right)<1$.

(iv) The triplet $\left(E, A, A_{d}\right)$ is said to be admissible if it is regular, causal and stable. 
Applying the controller (4) to the system (34), we have the closed-loop system

$$
E x(k+1)=\left(A+B K+H_{c} F_{\mathcal{c}}(k) G_{c}\right) x(k)+\left(A_{d}+H F(k) G_{d}\right) x(k-d)
$$

where

$$
H_{c}=\left[\begin{array}{ll}
H & \alpha B
\end{array}\right], F_{c}(k)=\operatorname{diag}[F(k) \Phi(k)], G_{c}=\left[\begin{array}{l}
G \\
K
\end{array}\right] .
$$

First, we consider the robust admissibility of the closed-loop system (35). In order to show a robust admissibility condition, we need the following theorem.

Theorem 5.2. (Xu E Lam [32]) (i) The descriptor delay system (34) with $u(k)=0$ and $\Delta A=$ $\Delta A_{d}=0$ is admissible if and only if there exist matrices $P>0, Q>0, X$ such that

$$
\left[\begin{array}{cc}
A^{T} P A-E^{T} P E+Q & A^{T} P A_{d} \\
A_{d}^{T} P A & A_{d}^{T} P A_{d}-Q
\end{array}\right]+\left[\begin{array}{c}
A^{T} \\
A_{d}^{T}
\end{array}\right] S X^{T}+X S^{T}\left[\begin{array}{c}
A^{T} \\
A_{d}^{T}
\end{array}\right]^{T}<0
$$

where $S \in \Re^{n \times(n-r)}$ is any matrix with full column rank and satisfies $E^{T} S=0$.

(ii) The descriptor delay system (34) with $u(k)=0$ and $\Delta A=\Delta A_{d}=0$ is admissible if and only if there exist matrices $P, Q>0$ such that

$$
\begin{array}{r}
E^{T} P E \geq 0, \\
{\left[\begin{array}{cc}
A^{T} P A-E^{T} P E+Q & A^{T} P A_{d} \\
A_{d}^{T} P A & A_{d}^{T} P A_{d}-Q
\end{array}\right]<0 .}
\end{array}
$$

Robust admissibility conditions for uncertain descriptor delay system (35) are given in the following theorems.

Theorem 5.3. Given $\gamma$ and $K$, the descriptor system (35) is robustly admissible if there exist matrices $P>0, Q>0, X=\left[\begin{array}{ll}X_{1}^{T} X_{2}^{T}\end{array}\right]^{T}$ and scalars $\varepsilon_{1}>0, \varepsilon_{2}>0$ such that

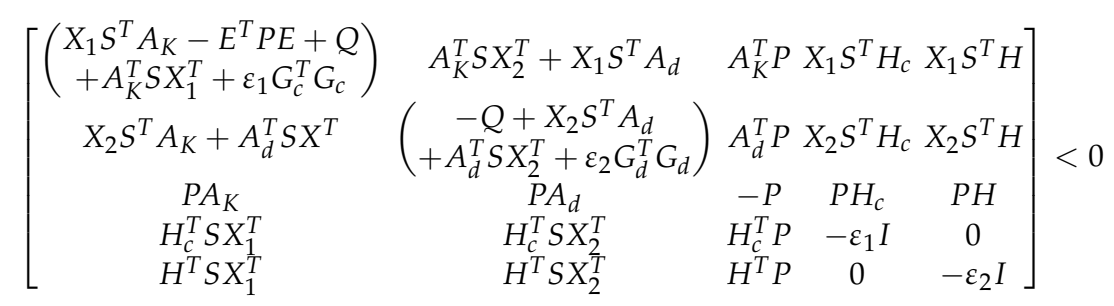

where $A_{K}=A+B K$ and $S \in \Re^{n \times(n-r)}$ is any matrix with full column rank and satisfies $E^{T} S=0$. 
Proof: Suppose that there exist matrices $P>0, Q>0, X=\left[\begin{array}{ll}X_{1}^{T} & X_{2}^{T}\end{array}\right]^{T}$ and scalars $\varepsilon_{1}>$ $0, \varepsilon_{2}>0$ such that the condition (39) holds. Then, by Schur complement formula, we have

$$
\begin{gathered}
{\left[\begin{array}{ccc}
X_{1} S^{T} A_{K}-E^{T} P E+A_{K}^{T} S X_{1}^{T}+Q & A_{K}^{T} S X_{2}^{T}+X_{1} S^{T} A_{d} & A_{K}^{T} P \\
X_{2} S^{T} A_{K}+A_{d}^{T} S X^{T} & -Q+X_{2} S^{T} A_{d}+A_{d}^{T} S X_{2}^{T} & A_{d}^{T} P \\
P A_{K} & P A_{d} & -P
\end{array}\right]} \\
+\varepsilon_{1}^{-1}\left[\begin{array}{c}
X_{1} S^{T} H_{c} \\
X_{2} S^{T} H_{c} \\
P H_{c}
\end{array}\right]\left[\begin{array}{lll}
H_{c}^{T} S X_{1}^{T} & H_{c}^{T} S X_{2}^{T} H_{c}^{T} P
\end{array}\right]+\varepsilon_{1}\left[\begin{array}{c}
G_{c}^{T} \\
0 \\
0
\end{array}\right]\left[\begin{array}{lll}
G_{c} & 0 & 0
\end{array}\right] \\
+\varepsilon_{2}^{-1}\left[\begin{array}{c}
X_{1} S^{T} H \\
X_{2} S^{T} H \\
P H
\end{array}\right]\left[\begin{array}{lll}
H^{T} S X_{1}^{T} & H^{T} S X_{2}^{T} & H^{T} P
\end{array}\right]+\varepsilon_{2}\left[\begin{array}{c}
0 \\
G_{d}^{T} \\
0
\end{array}\right]\left[\begin{array}{lll}
0 & G_{d} & 0
\end{array}\right]<0 .
\end{gathered}
$$

Now, using Lemma 3.2, we have

$$
\begin{aligned}
& \begin{array}{c}
{\left[\begin{array}{ccc}
\Delta_{11} & \Delta_{12} & \left(A_{K}+H_{c} F_{c}(k) G_{c}\right)^{T} P \\
\Delta_{12}^{T} & \Delta_{22} & \left(A_{d}+H F(k) G_{d}\right)^{T} P \\
P\left(A_{K}+H_{c} F_{c}(k) G_{c}\right) P\left(A_{d}+H F(k) G_{d}\right) & -P
\end{array}\right]} \\
{\left[\begin{array}{ccc}
X_{1} S^{T} A_{K}-E^{T} P E+A_{K}^{T} S X_{1}^{T}+Q & A_{K}^{T} S X_{2}^{T}+X_{1} S^{T} A_{d} & A_{K}^{T} P \\
X_{2} S^{T} A_{K}+A_{d}^{T} S X^{T} & -Q+X_{2} S^{T} A_{d}+A_{d}^{T} S X_{2}^{T} & A_{d}^{T} P \\
P A_{K} & P A_{d} & -P
\end{array}\right]}
\end{array} \\
& +\left[\begin{array}{c}
X_{1} S^{T} H_{c} \\
X_{2} S^{T} H_{c} \\
P H_{c}
\end{array}\right] F_{c}(k)\left[\begin{array}{lll}
G_{c} & 0 & 0
\end{array}\right]+\left[\begin{array}{c}
G_{c}^{T} \\
0 \\
0
\end{array}\right] F_{c}^{T}(k)\left[\begin{array}{lll}
H_{c}^{T} S X_{1}^{T} & H_{c}^{T} S X_{2}^{T} & H_{c}^{T} P
\end{array}\right] \\
& +\left[\begin{array}{c}
X_{1} S^{T} H_{c} \\
X_{2} S^{T} H_{c} \\
P H_{c}
\end{array}\right] F(k)\left[\begin{array}{lll}
0 & G_{d} & 0
\end{array}\right]+\left[\begin{array}{c}
0 \\
G_{d}^{T} \\
0
\end{array}\right] F^{T}(k)\left[\begin{array}{lll}
H_{c}^{T} S X_{1}^{T} & H_{c}^{T} S X_{2}^{T} & H_{c}^{T} P
\end{array}\right] \\
& \leq\left[\begin{array}{ccc}
X_{1} S^{T} A_{K}-E^{T} P E+A_{K}^{T} S X_{1}^{T}+Q & A_{K}^{T} S X_{2}^{T}+X_{1} S^{T} A_{d} & A_{K}^{T} P \\
X_{2} S^{T} A_{K}+A_{d}^{T} S X^{T} & -Q+X_{2} S^{T} A_{d}+A_{d}^{T} S X_{2}^{T} & A_{d}^{T} P \\
P A_{K} & P A_{d} & -P
\end{array}\right] \\
& +\varepsilon_{1}^{-1}\left[\begin{array}{c}
X_{1} S^{T} H_{c} \\
X_{2} S^{T} H_{c} \\
P H_{c} \\
0
\end{array}\right]\left[\begin{array}{lll}
H_{c}^{T} S X_{1}^{T} & H_{c}^{T} S X_{2}^{T} & H_{c}^{T} P
\end{array}\right]+\varepsilon_{1}\left[\begin{array}{c}
G_{c}^{T} \\
0 \\
0
\end{array}\right]\left[\begin{array}{lll}
G_{c} & 0 & 0
\end{array}\right] \\
& +\varepsilon_{2}^{-1}\left[\begin{array}{c}
X_{1} S^{T} H_{c} \\
X_{2} S^{T} H_{c} \\
P H_{c} \\
0
\end{array}\right]\left[\begin{array}{lll}
H_{c}^{T} S X_{1}^{T} H_{c}^{T} S X_{2}^{T} H_{c}^{T} P
\end{array}\right]+\varepsilon_{2}\left[\begin{array}{c}
0 \\
G_{d}^{T} \\
0
\end{array}\right]\left[\begin{array}{lll}
0 & G_{d} & 0
\end{array}\right]
\end{aligned}
$$

where

$$
\begin{aligned}
& \Delta_{11}=X_{1} S^{T}\left(A+B K+H_{c} F_{c}(k) G_{c}\right)+\left(A+B K+H_{c} F_{c}(k) G_{c}\right)^{T} S X_{1}^{T}-E^{T} P E+Q, \\
& \Delta_{12}=\left(A_{K}+H_{c} F_{c}(k) G_{c}\right)^{T} S X_{2}^{T}+X_{1} S^{T}\left(A_{d}+H F(k) G_{d}\right), \\
& \Delta_{22}=-Q+X_{2} S^{T}\left(A_{d}+H F(k) G_{d}\right)+\left(A_{d}+H F(k) G_{d}\right)^{T} S X_{2}^{T} .
\end{aligned}
$$


It follows from (40) that

$$
\left[\begin{array}{ccc}
\Delta_{11} & \Delta_{12} & \left(A_{K}+H_{c} F_{c}(k) G_{c}\right)^{T} P \\
\Delta_{12}^{T} & \Delta_{22} & \left(A_{d}+H F(k) G_{d}\right)^{T} P \\
P\left(A_{K}+H_{c} F_{c}(k) G_{c}\right) P\left(A_{d}+H F(k) G_{d}\right) & -P
\end{array}\right]<0 .
$$

This implies by Schur complement formula and Theorem 5.2(i) that the descriptor system (35) is robustly admissible.

Similarly, we can prove the following theorem by using Theorem 5.2(ii).

Theorem 5.4. Given $\gamma$ and $K$, the descriptor system (35) is robustly admissible if there exist matrices $P>0, Q>0$ and scalars $\varepsilon_{1}>0, \varepsilon_{2}>0$ such that (37), and

$$
\left[\begin{array}{ccccc}
-E^{T} P E+Q+\varepsilon_{1} G_{c}^{T} G_{c} & 0 & A_{K}^{T} P & 0 & 0 \\
0 & -Q+\varepsilon_{2} G_{d}^{T} G_{d} & A_{d}^{T} P & 0 & 0 \\
P A_{K} & P A_{d} & -P & P H_{c} & P H \\
0 & 0 & H_{c}^{T} P & -\varepsilon_{1} I & 0 \\
0 & 0 & H^{T} P & 0 & -\varepsilon_{2} I
\end{array}\right]<0
$$

where $A_{K}=A+B K$.

\subsection{Control design for time-delay systems}

Now, we are ready to propose control design methods for uncertain descriptor delay systems. The following theorems propose design methods of a non-fragile controller that makes the system (34) robustly admissible.

Theorem 5.5. There exists a controller (4) that makes the descriptor system (34) robustly admissible if there exist matrices $P>0, Q>0, X=\left[X_{1}^{T} X_{2}^{T}\right]^{T}$ and scalars $\varepsilon_{1}>0, \varepsilon_{2}>0$ such that

$$
\begin{array}{r}
\Gamma=P^{-1}-\varepsilon_{1}^{-1} H_{c} H_{c}^{T}-\varepsilon_{2}^{-1} H H^{T}>0, \\
W=Q-X_{2} S^{T} A_{d}-A_{d}^{T} S X_{2}^{T}-\varepsilon_{2} G_{d}^{T} G_{d}-\Theta_{D}^{T} \Gamma^{-1} \Theta_{D} \\
-X_{2} S^{T}\left(\varepsilon_{1}^{-1} H_{c} H_{c}^{T}+\varepsilon_{2}^{-1} H H^{T}\right) S X_{2}^{T}>0, \\
X_{1} S^{T} A+A^{T} S X_{1}^{T}-E^{T} P E+Q+\varepsilon_{1} G^{T} G+X_{1} S^{T}\left(\varepsilon_{1}^{-1} H_{c} H_{c}^{T}+\varepsilon_{2}^{-1} H H^{T}\right) S X_{1}^{T} \\
+\Theta_{A}^{T} \Gamma^{-1} \Theta_{A}+Y W^{-1} Y^{T}-\Psi \Lambda^{-1} \Psi^{T}<0
\end{array}
$$

where $S \in \Re^{n \times(n-r)}$ is any matrix with full column rank and satisfies $E^{T} S=0$, and

$$
\begin{aligned}
& \Psi=\left(X_{1} S^{T}+\Theta_{A}^{T} \Gamma^{-1}\right) B+Y W^{-1}\left(S X_{2}^{T}+\Theta_{D}^{T} \Gamma^{-1}\right) B, \\
& \Lambda=B^{T} \Gamma^{-1} B+\varepsilon_{1} I+B^{T}\left(S X_{2}^{T}+\Gamma^{-1} \Theta_{D}\right) W^{-1}\left(X_{2} S^{T}+\Theta_{D}^{T} \Gamma^{-1}\right) B, \\
& Y=A^{T} S X_{2}^{T}+\left(X_{1} S^{T}+\Theta_{A}^{T} \Gamma^{-1}\right) \Theta_{D}, \\
& \Theta_{A}=A+\left(\varepsilon_{1}^{-1} H_{c} H_{c}^{T}+\varepsilon_{2}^{-1} H H^{T}\right) S X_{1}^{T}, \\
& \Theta_{D}=A_{d}+\left(\varepsilon_{1}^{-1} H_{c} H_{c}^{T}+\varepsilon_{2}^{-1} H H^{T}\right) S X_{2}^{T} .
\end{aligned}
$$


In this case, a feedback gain in the controller (4) is given by

$$
K=-\Lambda^{-1} \Psi^{T}
$$

Proof: The closed-loop system (35) with the feedback gain (45) is given by

$$
E x(k+1)=\left(A-B \Lambda^{-1} \Psi^{T}+H_{c} F_{c}(k) G_{c}\right) x(k)+\left(A_{d}+H F(k) G_{d}\right) x(k-d)
$$

where $G_{c}=\left[G^{T}-\Psi \Lambda^{-1}\right]^{T}$. Then, by some mathematical manipulation, we have

$$
\begin{aligned}
& X_{1} S^{T}\left(A-B \Lambda^{-1} \Psi^{T}\right)+\left(A-B \Lambda^{-1} \Psi^{T}\right)^{T} S X_{1}^{T}+\varepsilon_{1} G_{c}^{T} G_{c} \\
= & X_{1} S^{T} A-X_{1} S^{T} B \Lambda^{-1} \Psi^{T}+A^{T} S X_{1}^{T}-\Psi \Lambda^{-1} B^{T} S X_{1}^{T}+\varepsilon_{1} G^{T} G+\varepsilon_{1} \Psi \Lambda^{-1} \Lambda^{-1} \Psi^{T}, \\
& {\left[A-B \Lambda^{-1} \Psi^{T}+\left(\varepsilon_{1}^{-1} H_{c} H_{c}^{T}+\varepsilon_{2}^{-1} H H^{T}\right) S X_{1}^{T}\right]^{T} \Gamma^{-1} } \\
& \times\left[A-B \Lambda^{-1} \Psi^{T}+\left(\varepsilon_{1}^{-1} H_{c} H_{c}^{T}+\varepsilon_{2}^{-1} H H^{T}\right) S X_{1}^{T}\right] \\
= & \Theta_{A}^{T} \Gamma^{-1} \Theta_{A}-\Psi \Lambda^{-1} B^{T} \Gamma^{-1} \Theta_{A}-\Theta_{A}^{T} \Gamma^{-1} B \Lambda^{-1} \Psi^{T}+\Psi \Lambda^{-1} B^{T} \Gamma^{-1} B \Lambda^{-1} \Psi^{T}, \\
& {\left[\left(A-B \Lambda^{-1} \Psi^{T}\right)^{T} S X_{2}^{T}+\left(X_{1} S^{T}+\left(A-B \Lambda^{-1} \Psi^{T}+\varepsilon_{1}^{-1} H_{c} H_{c}^{T} S X_{1}^{T}\right)^{T} \Gamma^{-1}\right) \Theta_{D}\right] W^{-1} } \\
& \left.\quad \times\left[\left(A-B \Lambda^{-1} \Psi^{T}\right)^{T} S X_{2}^{T}+\left(X_{1} S^{T}+\left(A-B \Lambda^{-1} \Psi^{T}+\varepsilon_{1}^{-1} H_{c} H_{c}^{T} S X_{1}^{T}\right)^{T} \Gamma^{-1}\right) \Theta_{D}\right)\right]^{T} \\
= & Y W^{-1} Y^{T}-\Psi \Lambda^{-1} B^{T}\left(S X_{2}^{T}+\Gamma^{-1} \Theta_{D}\right)^{T} W^{-1} Y^{T}-Y W^{-1}\left(S X_{2}^{T}+\Gamma^{-1} \Theta_{D}\right)^{T} B \Lambda^{-1} \Psi^{T} \\
& +\Psi \Lambda^{-1} B^{T}\left(S X_{2}^{T}+\Gamma^{-1} \Theta_{D}\right) W^{-1}\left(S X_{2}^{T}+\Gamma^{-1} \Theta_{D}\right)^{T} B \Lambda^{-1} \Psi^{T} .
\end{aligned}
$$

Thus, it can be verified with (44) that

$$
\begin{aligned}
& X_{1} S^{T}\left(A-B \Lambda^{-1} \Psi^{T}\right)+\left(A-B \Lambda^{-1} \Psi^{T}\right)^{T} S X_{1}^{T}+\varepsilon_{1} G_{c}^{T} G_{c}-E^{T} P E+Q \\
& +X_{1} S^{T}\left(\varepsilon_{1}^{-1} H_{c} H_{c}^{T}+\varepsilon_{2}^{-1} H H^{T}\right) S X_{1}^{T}+\left[A-B \Lambda^{-1} \Psi^{T}+\left(\varepsilon_{1}^{-1} H_{c} H_{c}^{T}+\varepsilon_{2}^{-1} H H^{T}\right) S X_{1}^{T}\right]^{T} \\
& \times \Gamma^{-1}\left[A-B \Lambda^{-1} \Psi^{T}+\left(\varepsilon_{1}^{-1} H_{c} H_{c}^{T}+\varepsilon_{2}^{-1} H H^{T}\right) S X_{1}^{T}\right] \\
& \left.+\left(A-B \Lambda^{-1} \Psi^{T}\right)^{T} S X_{2}^{T}+\left(X_{1} S^{T}+\left(A-B \Lambda^{-1} \Psi^{T}+\varepsilon_{1}^{-1} H_{c} H_{c}^{T} S X_{1}^{T}\right)^{T} \Gamma^{-1}\right) \Theta_{D}\right] W^{-1} \\
& \left.\quad \times\left[\left(A-B \Lambda^{-1} \Psi^{T}\right)^{T} S X_{2}^{T}+\left(X_{1} S^{T}+\left(A-B \Lambda^{-1} \Psi^{T}+\varepsilon_{1}^{-1} H_{c} H_{c}^{T} S X_{1}^{T}\right)^{T} \Gamma^{-1}\right) \Theta_{D}\right)\right]^{T} \\
= & X_{1} S^{T} A+A^{T} S X_{1}^{T}+\varepsilon_{1} G^{T} G-E^{T} P E+Q+X_{1} S^{T}\left(\varepsilon_{1}^{-1} H_{c} H_{c}^{T}+\varepsilon_{2}^{-1} H H^{T}\right) S X_{1}^{T} \\
& +\Theta_{A}^{T} \Gamma^{-1} \Theta_{A}+Y W^{-1} Y^{T}-\Psi \Lambda^{-1} \Psi^{T}<0 .
\end{aligned}
$$

By Schur complement formula, we obtain

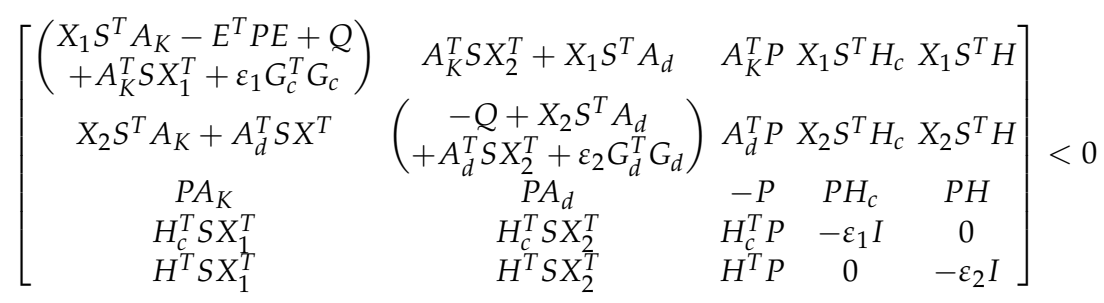


where $A_{K}=A-B \Lambda^{-1} \Psi^{T}$. Hence, by Theorem 5.3 we can show that the closed-loop system (35) is robustly admissible.

The following theorem can similarly be obtained from Theorem 5.4.

Theorem 5.6. There exists a controller (4) that makes the descriptor system (34) robustly admissible if there exist matrices $P>0, Q>0$ and scalars $\varepsilon_{1}>0, \varepsilon_{2}>0$ such that (37), (42),

$$
\begin{array}{r}
W=Q-\varepsilon_{2} G_{d}^{T} G_{d}-A_{d}^{T} \Gamma^{-1} A_{d}>0 \\
-E^{T} P E+Q+\varepsilon_{1} G^{T} G+A^{T} \Gamma^{-1} A+A^{T} \Gamma^{-1} A_{d} W^{-1} A_{d}^{T} \Gamma^{-1} A-\Psi \Lambda^{-1} \Psi^{T}<0
\end{array}
$$

where $\Gamma$ is given in (42), and

$$
\begin{aligned}
& \Psi=A^{T} \Gamma^{-1} B+A^{T} \Gamma^{-1} A_{d} W^{-1} A_{d}^{T} \Gamma^{-1} B \\
& \Lambda=B^{T} \Gamma^{-1} B+\varepsilon_{1} I+B^{T} \Gamma^{-1} A_{d} W^{-1} A_{d}^{T} \Gamma^{-1} B .
\end{aligned}
$$

In this case, a feedback gain in the controller (4) is given as in (45).

\section{Numerical examples}

In order to illustrate our control design methods, we consider the following two examples. The first one shows a non-fragile controller design method for an uncertain system, and the second gives the same class of a controller design method for a time-delay counterpart.

Consider an uncertain system:

$$
\left[\begin{array}{ll}
1 & 0 \\
0 & 0
\end{array}\right] x(k+1)=\left(\left[\begin{array}{ll}
0 & 1 \\
1 & 0.2
\end{array}\right]+\left[\begin{array}{l}
0.06 \\
0.08
\end{array}\right] F(k)[0.20]\right) x(k)+\left[\begin{array}{l}
0.2 \\
0.3
\end{array}\right] u(k) .
$$

Assuming the measure of non-fragility $\alpha=0.3$, we apply Theorem 3.7 or 3.8, which gives a non-fragile control gain $K$ in (4):

$$
K=[-4.0650-0.8131]
$$

Next, we consider a uncertain time-delay system

$$
\begin{aligned}
{\left[\begin{array}{ll}
1 & 0 \\
0 & 0
\end{array}\right] x(k+1)=} & \left(\left[\begin{array}{cc}
1 & 0.2 \\
0.1 & 0.3
\end{array}\right]+\left[\begin{array}{l}
0.06 \\
0.08
\end{array}\right] F(k)\left[\begin{array}{ll}
0.1 & 0
\end{array}\right]\right) x(k) \\
& +\left[\begin{array}{cc}
1 & 0.2 \\
0.1 & 0.3
\end{array}\right] x(k-3)+\left[\begin{array}{l}
0.1 \\
0.2
\end{array}\right] u(k) .
\end{aligned}
$$

Assuming the measure of non-fragility $\alpha=0.35$, we apply Theorem 5.5 or 5.6 , which gives a non-fragile control gain $K$ in (4):

$$
K=[-0.6349-1.9048]
$$




\section{Conclusions}

In this chapter, we investigated non-fragile control system analysis and design for uncertain discrete-time descriptor systems when the controller has some uncertainty in gain matrix. The controller is assumed to have multiplicative uncertainty in gain matrix. First, the robust admissibility of uncertain descriptor systems was discussed and the non-fragile control design methods were proposed. Then, theory was developed to the robust admissibility with $\mathrm{H}_{\infty}$ disturbance attenuation. Necessary and Sufficient conditions for the robust admissibility with $\mathrm{H}_{\infty}$ disturbance attenuation were obtained. Based on such conditions, the $\mathrm{H}_{\infty}$ non-fragile controller design methods were proposed. Next, uncertain descriptor systems with delay were considered. Based on system analysis of such systems, the non-fragile control design methods were proposed. Numerical examples were finally given to illustrate our controller design methods.

\section{Author details}

Jun Yoneyama, Yuzu Uchida and Ryutaro Takada

Department of Electronics and Electrical Engineering, College of Science and Engineering, Aoyama Gakuin University, Japan

\section{References}

[1] Boukas, E.K. \& Liu, Z.K. Delay-dependent stability analysis of singular linear continuous-time system, IEE Proceedings on Control Theory and Applications, 2003;150 325-330.

[2] Chen, S.-H. \& Chou, J.-H. Stability robustness of linear discrete singular time-delay systems with structured parameter uncertainties, IEE Proceedings on Control Theory and Applications, 2003;150 295-302.

[3] Chen, S.-J. \& Lin, J.-L. Robust stability of discrete time-delay uncertain singular systems, IEE Proceedings on Control Theory and Applications, 2004;151 45-52.

[4] Dai, L. Singular Control Systems, Springer, Berlin, Germany; 1981.

[5] Du, H.: Lam, J. and Sze, K. Y. Non-fragile output feedback $\mathrm{H}_{\infty}$ vehicle suspension control using genetic algorithm, Engineering Applications of Artificial Intelligence, 2003;16 667-680.

[6] Fridman, E. Stability of linear descriptor system with delay: a Lyapunov-based approach, Journal of Mathematical Analysis and Applications, 2002;273 24-44.

[7] Fridman, E. \& Shaked, U. $\mathrm{H}_{\infty}$-control of linear state-delay descriptor systems: an LMI approach, Linear Algebra and its Applications, 2002;351 271-302.

[8] Fridman, E. \& Shaked, U. Stability and guaranteed cost control of uncertain discrete delay systems, International Journal of Control, 2005;78 235-246. 
[9] Fridman, E. \& Shaked, U. Delay-dependent $\mathrm{H}_{\infty}$ control of uncertain discrete delay systems, European Journal of Control, 2005;11 29-37.

[10] Gao, H., Lam, J., Wang, C. \& Wang, Y. delay-dependent output feedback stabilization of discrete-time systems with time-varying state delay, IEE Proc. Control Theory Appl., 2004;151 691-698.

[11] Gao, H. \& Chen, T. New results on stability of discrete-time systems with time-varying state delay, IEEE Transactions on Automatic Control, 2007;52 328-334.

[12] Hara, M. \& Yoneyama, J. New robust stability condition for uncertain discrete-time systems with time-varying delay, in SICE Annual Conference 2008, 2008;743-747, Tokyo, August 2008.

[13] Hara, M. \& Yoneyama, J. An improved robust stability condition for uncertain discrete time-varying delay systems, Journal of Cybernetics and Systems, 2009;2 23-27.

[14] He, Y., Wang, Q., Xie, L. \& Lin, C. Further improvement of free-weighting matrices technique for systems with time-varying delay, IEEE Transactions on Automatic Control, 2007;52 293-299.

[15] Hou, Y., Liao, T., Yan, J. \& Lien, C. Non-fragile $\mathrm{H}_{\infty}$ control for singular systems with state and input time-varying delays, International Journal of Nonlinear Sciences and Numerical Simulation, 2007;8 31-40.

[16] Kim, J. \& Oh, D.-C. Robust and non-fragile $\mathrm{H}_{\infty}$ control for descriptor systems with parameter uncertainties and time delay, International Journal of Control, Automation, and Systems, 2007;5 8-14.

[17] Lin, C., Lam, J., Wang, J.L. \& Yang, G.H. Analysis of robust stability for interval descriptor system, Systems and Control Letters, 2001;42 267-278.

[18] Li, X. \& de Souza, C. E. Delay dependent robust stability and stabilization of uncertain linear delay systems: a linear matrix inequality approach, IEEE Transactions on Automatic Control, 1997;42 1144-1148.

[19] Lien, C. Non-fragile guaranteed cost control for uncertain neutral dynamic systems with time-varying delays in state and control input, Chaos, Solitons and Fractals, 2007;31 889-899.

[20] Lien, C. $\mathrm{H}_{\infty}$ non-fragile observer-based controls of dynamical systems via LMI optimization approach, Chaos, Solitons and Fractals, 2007;34 428-436.

[21] Lien, C. Cheng, W. Tsai, C. \& Yu, K. Non-fragile observer-based controls of linear system via LMI approach, Chaos, Solitons and Fractals, 2007;32 1530-1537.

[22] Lu, G. \& Ho, D.W.C. Generalized quadratic stability for continuous-time singular systems with nonlinear perturbation, IEEE Transactions on Automatic Control, 2006;51 818-823. 
[23] Lv, L. \& Lin, Z. Analysis and design of singular linear systems under actuator saturation and disturbances, Systems and Control Letters, 2008;57 904-912.

[24] Ma, S., Cheng, Z. \& Zhang, C. Delay-dependent robust stability and stabilisation for uncertain discrete singular systems with time-varying delays, IEE Proceedings on Control Theory and Applications, 2007;1 1086-1095.

[25] Ma, S., Zhang, C. \& Cheng, Z. Delay-dependent Robust $\mathrm{H}_{\infty}$ Control for Uncertain Discrete-Time Singular Systems with Time-Delays, Journal of Computational and Applied Mathematics, 2008;217 194-211.

[26] Mahmoud, M.S. Robust Control and Filtering for Time-Delay Systems, New York: Marcel Dekker, Inc; 2000.

[27] Palhares, R.M., Campos, C.D., Ekel, P. Ya., Leles, M.C.R. \& D'Angelo, M.F.S.V. Delay-dependent robust $\mathrm{H}_{\infty}$ control of uncertain linear systems with lumped delays, IEE Proc. Control Theory Appl., 2005;152 27-33

[28] Wo, S., Zou, Y., Chen, Q., \& Xu, S. Non-fragile controller design for discrete descriptor systems, Journal of the Franklin Institute, 2009;346 914-922.

[29] Xie, L. Output Feedback $H_{\infty}$ Control of systems with parameter uncertainty, International Journal of Control, 1996;63, 741-750.

[30] Xie, L. \& de Souza, C.E. Robust $\mathrm{H}_{\infty}$ control for linear systems with norm-bounded time-varying uncertainty, IEEE Transactions on Automatic Control, 1992;37 1188-1191.

[31] Xu, S., Van Dooren, P., Stefan, R. and Lam, J. Robust stability and stabilization for singular systems with state delay and parameter uncertainty, IEEE Transactions on Automatic Control, 2002;47 1122-1128.

[32] Xu, S. \& Lam, J. Robust Control and Filtering of Singular Systems, Springer-Verlag Berlin Heidelberg; 2006.

[33] Xu, S., Lam, J. \& Zou, Y. Improved conditions for delay-dependent robust stability and stabilization of uncertain discrete-time systems, Asian Journal of Control, 2005;7 344-348.

[34] Xu, S., Lam, J. \& Zou, Y. New results on delay-dependent robust $\mathrm{H}_{\infty}$ control for systems with time-varying delays, Automatica, 2006;42 343-348.

[35] Ye, D. \& Yang, G. H. Adaptive robust $\mathrm{H}_{\infty}$ state feedback control for linear uncertain systems with time-varying delay, International Journal of Adaptive Control and Signal Processing, 2008;22 845-858.

[36] Yoneyama, J. \& Tsuchiya, T. New delay-dependent conditions on robust stability and stabilisation for discrete-time systems with time-delay, International Journal of Systems Science, 2008;39 1033-1040. 
[37] Zhang, X.-M. \& Han, Q.-L. A new finite sum inequality approach to delay-dependent $\mathrm{H}_{\infty}$ control of discrete-time systems with time-varying delay, International Journal of Robust and Nonlinear Control, 2008;18 630-647. 